\title{
Bêta-thalassémie majeure : actualisation de la prise en charge en médecine bucco-dentaire
}

\author{
Sarah Millot ${ }^{1,2,3, \star}$, Henri Woimant ${ }^{2}$, Anne-Laure Ejeil ${ }^{1,3}$, Jean-Luc Charrier ${ }^{1,3}$ \\ 1 Faculté d'Odontologie, Université Paris Descartes, Paris, France \\ 2 Service de Stomatologie, Hôpital Saint Antoine, Paris, France \\ 3 Service d'Odontologie, Hôpital Bretonneau, Paris, France
}

(Reçu le 11 novembre 2010, accepté le $1^{\text {er }}$ décembre 2010)

Mots clés :

thalassémie majeure /

ostéoporose /

bisphosphonates /

infection

\begin{abstract}
Résumé - La $\beta$-thalassémie majeure représente un syndrome génétique qui s'accompagne d'un défaut de synthèse de l'hémoglobine et entraine une anémie sévère. Malgré des progrès importants dans la prise en charge des patients, il existe toujours de nombreuses complications dues à la maladie elle-même, aux transfusions répétées, à la surcharge en fer et à la toxicité des chélateurs du fer; elles dominent encore le pronostic vital. Toutes ces complications et/ou leurs traitements ont un impact sur la prise en charge bucco-dentaire des patients; le cas rapporté en constitue un exemple. Tout praticien peut rencontrer dans sa pratique un patient atteint d'une $\beta$-thalassémie majeure : il est indispensable de connaitre les mesures particulières qui doivent être prises lors de l'anamnèse, de l'examen clinique, des interventions chirurgicales et de la surveillance postopératoire.
\end{abstract}

\begin{abstract}
- $\beta$-thalassemia major: news repercussions of this pathology and clinical managment in oral surgery. Beta-thalassemias major are hereditary disorders resulting from defects in hemoglobin production and lead to severe anemia. Despite major advances in clinical management, several complications due to disease, lifelong transfusions, iron overload, iron chelation toxicity persist and still influence the prognosis. Those complications and/or their treatments have oral repercussions; here we present an example. Clinicians may encounter patients with beta-thalassemias in daily practice and it is essential to know the precautions that should be taken during medical history, oral examination, oral surgery and during recovery after surgery.
\end{abstract}

Les ß-thalassémies sont des maladies génétiques, à transmission généralement autosomique récessive, résultant d'une insuffisance de production des chaines de ß-globine [1]. Ces hémoglobinopathies sont très répandues dans le bassin méditerranéen mais leur distribution tend à devenir ubiquitaire en raison du mouvement des populations. On différencie la ß-thalassémie hétérozygote ou mineure de la thalassémie homozygote ou thalassémie majeure ( $\beta$-TM). Le tableau clinique de la ß-thalassémie est variable : elle peut être asymptomatique ou rapidement létale pour les formes les plus sévères. Les chaines de globine alpha en excès précipitent et leurs produits de dégradation dans les globules rouges sont à l'origine d'une érythropoïèse inefficace et d'une destruction des globules rouges dans la circulation sanguine. Dans la $\beta$-TM, la première manifestation clinique est l'apparition d'une anémie sévère et précoce. Cette anémie stimule la production

\footnotetext{
*Correspondance : sarahmillot@yahoo.fr
}

d'érythropoiétine, responsable d'une prolifération massive de précurseurs de globules rouges dans la moelle osseuse; cette stimulation de la moelle osseuse entraine des déformations osseuses touchant notamment les os de la tête et de la face [2].

Actuellement la seule thérapeutique curative de la $\beta$-TM est la greffe de cellules souches hématopoïétiques mais seule une minorité de patients peut en bénéficier [3]. Le traitement conventionnel de la $\beta$-TM comporte des transfusions sanguines répétées, toutes les 3 à 5 semaines. Il en résulte une surcharge en fer qui représente la principale morbidité [4]. Un chélateur du fer est donc toujours associé au support transfusionnel.

Si les progrès considérables réalisés dans la prise en charge de ces patients ont permis une augmentation significative de l'espérance de vie, il n'en demeure pas moins des complications gravissimes, pour la plupart liées à la surcharge 
en fer [5]. Ces complications doivent être identifiées lors de la prise en charge bucco-dentaire hospitalière des patients thalassémiques. Nous avons donc un rôle dans le suivi de ces patients, dans l'éradication des foyers infectieux potentiellement gravissimes chez des sujets souvent fragilisés, et des précautions à prendre avant tout acte chirurgical en raison des risques liés éventuellement à leurs traitements (par exemple, traitement par bisphosphonates). Les recommandations sur le suivi des TM éditées en juin 2008 par Haute Autorité de Santé (HAS) insistent sur la nécessité d'une prise en charge multidisciplinaire [6].

\section{Observation}

Une patiente de 20 ans est adressée dans le Service de Stomatologie par le Service de Maladies infectieuses pour un syndrome inflammatoire avec hyperthermie $\left(40,2{ }^{\circ} \mathrm{C}\right)$, douleurs cervicales, adénopathies sous-angulo-mandibulaires droites et asthénie. L'anamnèse révèle une $\beta$-TM ayant nécessité une splénectomie à l'âge de 8 ans afin de réduire les apports transfusionnels. Une hémochromatose posttransfusionnelle est présente depuis 7 ans; elle est traitée par Ferriprox ${ }^{\circledR}$. La difficulté et la lourdeur de ce traitement chélateur ont amené la patiente à l'abandonner depuis 3 mois. Lors de son hospitalisation, en raison du risque infectieux lié à la splénectomie, un traitement associant amoxicilline et acide clavulanique par voie IV a été prescrit.

Lors de l'examen exo-buccal, on note un visage de forme carrée, un élargissement de l'arête nasale et une tuméfaction génienne droite douloureuse à la palpation. L'examen endobuccal révèle une tuméfaction vestibulaire fluctuante à hauteur de la 47, douloureuse à la palpation. La 36 a été extraite récemment et de nombreux soins conservateurs sont prévus, en particulier sur la 37. La muqueuse buccale ne présente pas d'anomalies mais on note une pâleur importante, surtout sur la face interne des joues. L'examen occlusal montre une relation molaire de classe II. La radiographie panoramique confirme la présence d'un kyste péri-apical sur la 47 (Fig. 1). L'extraction de la 47 est réalisée lors de la première consultation et on procède, dans le même temps, au curetage du kyste.

Les examens sanguins, effectués dès l'admission, confirment l'existence d'un syndrome inflammatoire et infectieux (hyperleucocytose et CRP très augmentée), une anémie sévère (hémoglobine : $6,8 \mathrm{~g} \cdot \mathrm{dL}^{-1}$ ) et une surcharge en fer importante objectivée par la concentration importante de la ferritine dans le sérum (Tab. I).

Après une semaine d'hospitalisation les anomalies biologiques persistent; pendant les 4 semaines d'hospitalisation, deux transfusions ont été nécessaires pour compenser partiellement l'anémie (hémoglobine : $9,5 \mathrm{~g} \cdot \mathrm{dL}^{-1}$ ) et faire régresser le syndrome infectieux. On peut noter que certaines constantes biologiques restent modifiées chez cette jeune femme, en raison de son affection et des nombreuses transfusions : thrombocytose et transaminases élevées. Pour le Service de Maladies infectieuses, il s'agissait d'un «syndrome

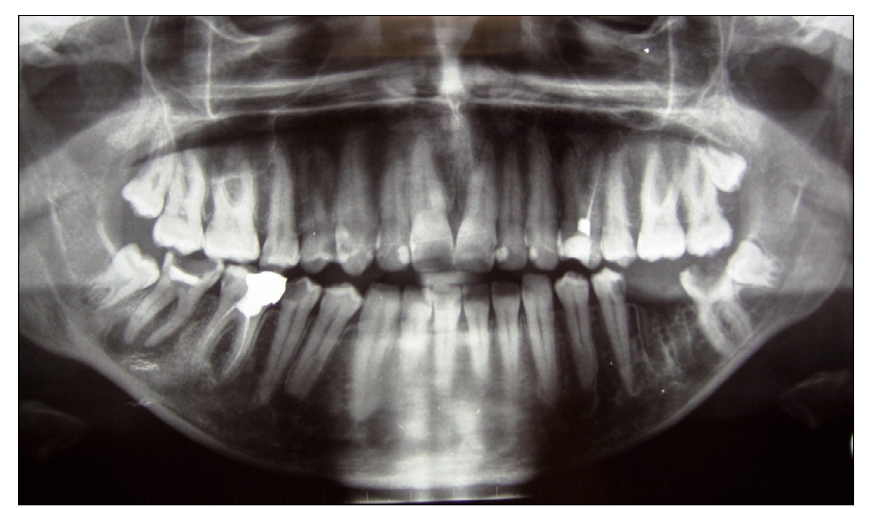

Fig. 1. Sur l'orthopantomogramme, on note une image apicale radiotransparente sur la 47 et une carie délabrante sur la 37.

Fig. 1. Orthopantomogramm shows an apical radiolucency on 47 and a destructive decay on 37.

Tableau I. Paramètres biologiques au début, pendant et à la fin de l'hospitalisation.

Table I. Biological date at the beginning, during and at the end of the hospitalization.

\begin{tabular}{|l|c|c|c|}
\hline Examens & Admission J0 & Hospitalisation J7 & Sortie J28 \\
\hline Hémoglobine $\left(\mathrm{g} \cdot \mathrm{dL}^{-1}\right)$ & 6,8 & 8,0 & 9,5 \\
\hline Leucocytes $\left(10^{9} \cdot \mathrm{L}^{-1}\right)$ & 26,26 & 15,59 & 7,91 \\
\hline Plaquettes $\left(\mathrm{mm}^{-3}\right)$ & 936000 & 514000 & 648000 \\
\hline CRP $\left(\mathrm{mg} \cdot \mathrm{L}^{-1}\right)$ & 31 & 9,7 & 3,3 \\
\hline ASAT $\left(\mathrm{UI} \cdot \mathrm{L}^{-1}\right)$ & 133 & 107 & 127 \\
\hline Ferritine $\left(\mathrm{ng} \cdot \mathrm{mL}^{-1}\right)$ & 11407 & - & - \\
\hline
\end{tabular}

infectieux chez une patiente thalassémique, splénectomisée, à point d'appel dentaire ».

\section{Commentaires}

Il semble bon de revenir sur quelques complications majeures, certaines très récentes, ayant chacune un impact en chirurgie buccale et qui nécessitent des mesures adéquates.

La surcharge en fer posttransfusionnelle représente la principale complication des $\beta$-TM. Elle domine le pronostic vital et touche quasiment tous les patients malgré le traitement avec un chélateur du fer. Les différents chélateurs sont dans l'ensemble mal tolérés; on est toujours à la recherche d'un chélateur bien supporté, favorisant ainsi une meilleure compliance. Le suivi biologique régulier de la ferritinémie représente l'élément le plus utilisé pour évaluer l'importance de la surcharge en fer [7]. Dans le cas rapporté, à l'admission, la ferritinémie est d'environ $11000 \mathrm{ng} \cdot \mathrm{mL}^{-1}$, soit plus de 100 fois la concentration normale; la patiente présente donc une surcharge en fer. Cet examen n'a pas été répété au cours de l'hospitalisation.

Cette accumulation de fer touche plusieurs tissus et, pour chacun des organes atteints, le praticien en chirurgie buccale peut être confronté à d'éventuelles complications nécessitant 
des mesures spécifiques. L'atteinte cardiaque est responsable des $2 / 3$ des décès chez les patients souffrant de $\beta$-TM par insuffisance cardiaque congestive, mort subite ou troubles du rythme $[5,8]$. Le traitement de la surcharge cardiaque comporte deux volets : une augmentation du traitement avec un chélateur du fer par voie IV continue et un traitement symptomatique. Ce dernier peut associer des vasodilatateurs, des diurétiques, des anti-arythmiques, des antivitamines $\mathrm{K}$ ou encore de la Digoxine ${ }^{\circledR}$. Il est fondamental de connaitre les traitements en cours puisqu'ils peuvent constituer une contreindication relative à l'utilisation des vasoconstricteurs lors de l'analgésie locale et nécessiter la prise en charge du risque hémorragique chez les patients sous antivitamines $K[9,10]$. La surcharge en fer peut également être responsable d'une atteinte hépatique se traduisant initialement par une fibrose, évoluant vers la cirrhose chez $10 \%$ des patients thalassémiques. L'incidence du carcinome hépato-cellulaire est plus élevée chez les patients atteints de $\beta$-TM. Cette atteinte hépatique s'accompagne d'une élévation des transaminases, anomalie biologique persistante chez cette patiente (Tab. I) [11]. Il peut également exister des complications endocriniennes, notamment un diabète insulino-dépendant chez 10 à $15 \%$ des patients.

Les patients thalassémiques splénectomisés présentent un risque infectieux plus élevé d'où la nécessité de proposer l'élimination des foyers infectieux bucco-dentaires sous couverture antibiotique. Après la splénectomie, les patients sont suivis pour les différentes vaccinations et la prescription d'antibiotiques de façon préventive [12]. Le contrôle de l'hygiène et de l'état bucco-dentaires est particulièrement important. Les patients splénectomisés ont également un risque plus élevé d'accidents thromboemboliques; certains sont traités par antivitamines K [13]. Les accidents thromboemboliques sont favorisés par les propriétés procoagulantes des globules rouges et la thrombocytose. Dans le cas rapporté, la patiente avait été splénectomisée à l'âge de 8 ans et il existait une thrombocytose (entre 500000 et 900000 plaquettes. $\mathrm{mm}^{-3}$ au cours de son hospitalisation).

L'atteinte osseuse constitue la troisième complication; elle est constante et représente une cause majeure de morbidité. Son étiologie est multifactorielle mais elle est principalement due à la prolifération importante de la moelle osseuse, aux déficits endocriniens et à la surcharge en fer. L'atteinte osseuse se traduit par deux affections distinctes : une hyperplasie de la moelle et une ostéoporose. La prolifération massive des précurseurs érythroïdes dans la moelle osseuse est à l'origine de déformations du squelette, notamment des os du crâne où, dans les cas typiques, on peut observer les « traits osseux thalassémiques caractéristiques » : aspect en "poils de brosse » sur les clichés du crâne de profil, maxillaires déformés à l'origine de malpositions dentaires et amincissement important des corticales [14-16]. Souvent il existe une rétrognathie mandibulaire. Dans le cas rapporté, le traitement précoce par transfusions a limité le développement des anomalies squelettiques. Malgré l'amélioration de la prise en charge (traitement hormonal substitutif, administration de calcium et de vitamine $D$, normalisation du taux d'hémoglobine), 70 à
$80 \%$ patients thalassémiques (adultes mais aussi enfants) présentent une perte de masse osseuse à l'origine de douleurs importantes et de fractures invalidantes [17]. Le traitement de cette ostéoporose constitue une priorité, et elle fait actuellement l'objet de nombreuses études. L'étude de Voskaridou et al. a confirmé l'efficacité du traitement par l'Aredia ${ }^{\circledR}$ (30 mg de pamidronate en perfusion intraveineuse par mois pendant 12 mois) [18]. Les bisphosphonates occupent actuellement une place importante dans la prise en charge de l'ostéoporose secondaire à la $\beta$-TM : ils permettent d'obtenir une amélioration significative de la densité osseuse et une régression des douleurs [19]. Des études sont nécessaires pour préciser la posologie et la molécule les mieux adaptées.

Les praticiens en médecine bucco-dentaire ont été récemment sensibilisés à la prise en charge particulière de patients traités ou allant être traités par bisphosphonates oraux pour une ostéoporose le plus souvent postménopausique. La $\beta$-TM constitue une nouvelle indication des bisphosphonates : les patients thalassémiques sont plus jeunes - moyenne d'âge 35,5 ans - [87] et se rencontrent autant chez les hommes que chez les femmes. Habituellement, ces patients ne sont pas concernés par un interrogatoire spécifique sur l'ostéoporose. On doit intégrer ces nouvelles données dans l'anamnèse et les protocoles en chirurgie buccale.

\section{Conclusion}

La $\beta$-TM est une pathologie rare mais grave. L'intérêt du cas rapporté réside dans l'évolution sévère d'un accident infectieux d'origine dentaire normalement banal. Ceci permet de rappeler les complications observées dans la $\beta$-TM. En médecine bucco-dentaire, l'intérêt se portait jadis sur les déformations osseuses de l'extrémité céphalique et les malpositions dentaires. Les progrès thérapeutiques ont profondément modifié le taux de survie des patients atteints de $\beta$-TM mais ces derniers restent fragiles et les traitements comportent des effets indésirables parfois fatals (en particulier la surcharge en fer secondaire aux transfusions sanguines).

Le cas rapporté illustre la nécessité d'un suivi régulier pour ces patients et l'importance du maintien d'un état buccodentaire satisfaisant car les foyers infectieux peuvent avoir des répercussions systémiques difficiles à contrôler, nécessitant parfois une longue hospitalisation. Ce cas permet également de faire le point sur les éventuelles difficultés de la prise en charge de ces patients dans différents domaines : risque infectieux, traitements en cours, troubles de la coagulation, risques liés aux bisphosphonates. Ces patients requièrent une prise en charge multidisciplinaire et une coordination entre les différents spécialistes de santé.

Crédits de recherche fournis par l'ARC (Association pour la Recherche sur le Cancer) et la SFH (Société française d'Hématologie).

Remerciements à l'Unité des Maladies génétiques du globule rouge, CHU Henri Mondor, Créteil, France

Conflits d'intérêt : aucun 


\section{Références}

1. Higgs DR, Thein SL, Woods WG. The molecular pathology of the thalassemias. In: The thalassemia syndromes (pp. 133-91), $4^{\text {th }}$ ed. Blackwell Science, Oxford, 2001.

2. Pootrakul $P$, Sirankapracha $P$, Hemsorach S. A correlation of erythrokinetics, ineffective erythropoiesis, and erythroid precursor apoptosis in Thai patients with thalassemia. Blood 2000;96:2606-12.

3. Rund D, Rachmilewitz E. Beta-thalassemia. N Engl J Med 2005;353:1135-46.

4. Lucarelli G, Andreani M, Angelucci E. The cure of thalassemia by bone marrow transplantation.Blood Rev 2002;16:81-5.

5. Cunningham MJ, Macklin EA, Cohen NA. Complications of $\beta$-thalassemia major in north America. Blood 2004;104:34-9.

6. Syndromes thalassémiques majeurs et intermédiaires: protocole national de diagnostic et de soins pour une maladie rare. Haute Autorité de Santé, Guide affection longue durée, 2008.

7. Oliveri NF, Brittenham GM. Iron-chelating therapy and the treatment of thalassemia. Blood 1997;89:739-61.

8. Borgna-Pignatti C, Cappellini MD, de Stefano P, Del Vecchio GC, Forni GL, Gamberini MR, Ghilardi R, Piga A, Romeo MA, Zhao $H$, Cnaan A. Cardiac morbidity and mortality in deferoxamine or deferiprone treated patients with thalassemia major. Blood 2006;1;107:3733-7.

9. Emploi des vasoconstricteurs en odonto-stomatologie. Recommandations. Med Buccale Chir Buccale 2003;9;2:65-94.

10. Prise en charge des patients sous traitements anti-vitamine $\mathrm{K}$ en chirurgie bucco-dentaire. Recommandations. Med Buccale Chir Buccale 2006;12:187-212.
11. Fragatou S, Tsourveloudis I, Manesis G. Incidence of hepatocellular carcinoma in a thalassemia unit. Hemoglobin 2010;34:221-6.

12. Cadili A, Gara C. Complications of splenectomy. Am J Med 2008;121:371-5.

13. Cappellini MD, Robbiolo L, Bottasso BM, Coppola R, Fiorelli G, Mannucci PM. Venous thromboembolism and hypercoagulability in splenectomized patients with thalassemia intermedia. $\mathrm{Br} \mathrm{J}$ Haematol 2000;111:467-73.

14. Jung S, Garret-Bernardin A, Grollemund B, Cojean N, Lutz P, Manière MC, Féki A. Manifestations dento-maxillo-faciales chez un enfant atteint d'une béta-thalassémie majeure traitée par greffe de cellules souches hématopoïétiques. A propos d'un cas. Med Buccale Chir Buccale 2006;12;3:163-70.

15. Van Dis M, Langlais R. The thalassemias : oral manifestations and complications. Oral Surg Oral Med Oral Pathol 1986;62:229-33.

16. Basu S, Kumar A. Hair-on-end appearance in radiograph of skull and facial bones in a case of beta thalassemia. $\mathrm{Br} \mathrm{J}$ Haematol 2008;144:807.

17. Chatterjee R, Bajoria R. Osteopenia-osteoporosis syndrome in patients with thalassemia: understanding of type of bone disease and response to treatment. Hemoglobin 2009;33 suppl 1:S136-8.

18. Voskaridou E, Terpos, Spina G, Palermos J, Rahemtulla A, Loutradi A Loukopoulos D. Pamidronate is an effective treatment for osteoporosis in patients with beta-thalassemia. $\mathrm{Br} \mathrm{J}$ Haematol 2003;123:730-7.

19. Leung TF, Chu Y, Lee V, Leung WK, Shing MM, Li CK. Long-term effects of pamidronate in thalassemic patients with severe bone mineral density deficits. Hemaglobin 2009;33:361-9. 